\title{
Three experiments to support the design of lightweight comfortable vehicle seats
}

\author{
P. Vink ${ }^{a}$, M. Franz ${ }^{b}$, I. Kamp ${ }^{a, b}$ and R. Zenk ${ }^{b}$ \\ ${ }^{a}$ Faculty of Industrial Design Engineering, Delft University of Technology, Landbergstraat 15, 2628 CE Delft, \\ The Netherlands \\ ${ }^{\mathrm{b}}$ BMW AG, Knorrstrasse 147, D-80788 München, Germany
}

\begin{abstract}
Seats need to be more lightweight for airplanes, cars, busses and even trains to contribute to a better environment and to reduce energy consumption. However, a reduction in comfort due to weight reduction is not preferable, which opens a new area of research: improving comfort with a minimum of material or with lightweight materials and systems. In this paper three experiments are performed to test the effects of light weight seats and parts of a seat on comfort. The first experiment shows that a new developed light weight massage system improves comfort and reduces muscle activity. The second experiment shows that the automatic seat adjustment without motors improves the comfort as well. The third experiment showed that a light weight seat following closely the human body contour is experienced on many aspects in the same way as current more heavy seats. More research and models will be needed in this ergonomic field which needs more attention.
\end{abstract}

Keywords: seat, comfort, sustainability, light weight, end user experience

\section{Introduction}

High fuel prizes demand for lighter vehicles. Another reason to develop vehicles with less and lighter materials is to achieve a more responsible image and meet the regulations. Seats contribute to the weight of airplanes, cars, busses and even trains and lighter materials or new designs are preferred for seats as well [4].

Of course the safety demands should still be met, but additionally, vehicle seat users demand at least the comfort they are used to according to Vink \& Hallbeck [6]. This could be contradictory as the current seats could be made more comfortable by adding weight. It is more problematic to improve comfort and reduce weight at the same time. Therefore, in the development programs of the Delft University of Technology and BMW where "sustainable wellbeing" is part of the vision, innovative seat concepts are developed that reduce weight. However, the question is whether these new concepts are appreciated by end users.

In this paper three concepts that are focused on lightweight and comfort level will be evaluated. End

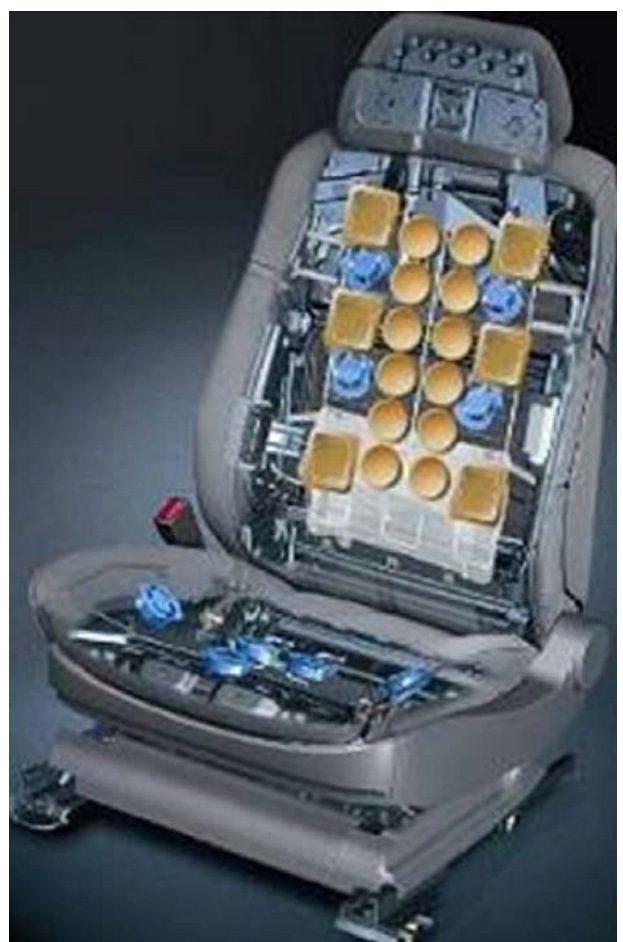

Fig. 1. The inflatable bladders in the seat used in experiment 1 . 
user opinions and appreciation will be studied to give input into design directions.

The first innovation is a lightweight massage system (LWMAS) for the backrest of a car seat (see Figure 1). This system does not use mechanical motors, but it uses airbladders that are inflated at different times. Including the pump the weight is 740 gram, which is much lower than the current systems [3]. An important aim of the massage was to rotate the vertebrae alternately by movement actuators. According to Kyung and Nussbaum [5] rotation between the vertebrae can have positive effects on the musculoskeletal system. Therefore, the system was built in such a way that parts of the back cushion inflate unevenly on both sides of the spine.

For example, this means that at Th3 level and at L3 level, there is inflation on the left side and, at Th9, inflation on the right side. This is reversed after a few seconds: Th3 and L3 are inflated on the right side and Th9 is inflated at the left side. Additionally, a vertical movement is created by pumping up the two bladders close to the spine followed by two others close to the previous two bladders. Several prototypes were needed to get a final working system, which is now patented worldwide. One possible disadvantage of such a lightweight system could be that the effects may be too small and might not be noticed by the passenger or driver. Therefore, an experiment was carried out containing questions where participants are asked whether they notice any effect that would indicate that the massage system has exceeded the threshold, in addition to their comfort experience. Also, electromyograms (EMG) were used to determine whether a massage system had a significant effect on muscle activity.

The second concept was a seat that can be adapted to the human body without using heavy mechanical seat adjustment motors. Sensors were added to a system of inflatable cushions and these inflatable cushions were used to automatically adjust the form of a seat by inflating or deflating different parts in the seat and back rest to enable a comfortable ('ideal') position defined by Zenk et al. [8]. This ideal pressure distribution was achieved as the sensors record the pressure. The algorithm of pumpibing the right bladders was developed in an extensive research program of the prof H. Bubb of the TU München [7]. The test car of this experiment was a BMW 7-Series, equipped with a multifunctional leather seat. It is possible to adjust the seat in 16 degrees of freedom allowing the ideal load distribution to be achieved. Zenk [7] presented in his $\mathrm{PhD}$ thesis that subjects preferred the position calculated by the seat based on the ideal pressure distribution above seat adjustments the drivers made themselves. However, there was no evidence yet that the human body is influenced. Therefore, in this study we measured the pressure in the intervertebral disc in several positions of the seat including the ideal one.

The third innovation concerned a light weight seat that consists only of a seat shell with inflatable cushions covered with a MESH material which distributes heat and humidity and a texture. It is important that the seat shell follows closely the human body contour that is in contact with the seat. Therefore, an experiment was done to determine the body contours, which were 3D scanned [2] of a group of drivers varying from $\mathrm{P} 5$ to $\mathrm{P} 95$. Based on these contours a $3 \mathrm{D}$ seat shell form was developed in CATIA (see Figure 2 ). In the working model based on this contour an inflatable cushion fills the gaps between the largest and smallest persons. To see how passengers and drivers react on this seat, a comparison of experience was made with two other seats.

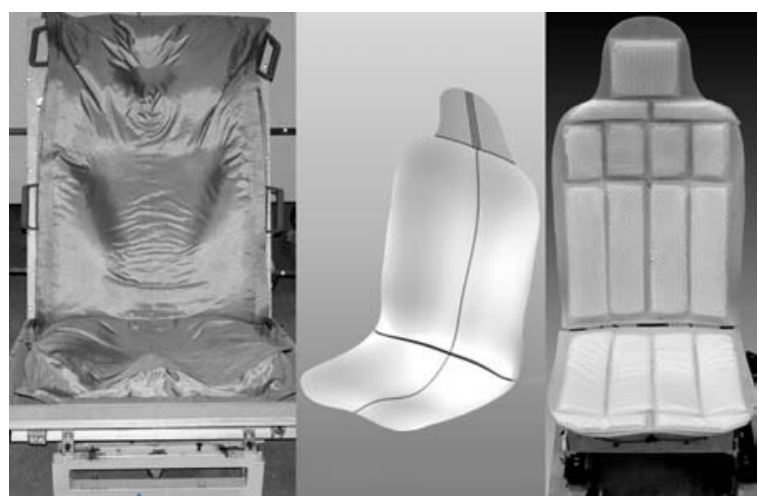

Fig. 2. Three stages in the seat development of the light weight seat: measuring the human contour (left), the CATIA $3 \mathrm{~d}$ model (middle), the seat shell with inflatable cushions (right).

\section{Methods}

Experiment 1: Twenty four BMW employees participated in testing the effect of the light weight massage seat. At least one 5th-percentile female length and one 95th-percentile male length participated in each experiment (mean \pm SD age $28.4 \pm 12.1$ years; mean $\pm \mathrm{SD}$ body height, $1.85 \pm 0.06 \mathrm{~m}$; mean $\pm \mathrm{SD}$ body weight, $80.1 \pm 8.2 \mathrm{~kg}$ ). 21 male and 3 female subjects volunteered to drive on a test track while EMG was recorded, and they also answered a questionnaire in each condition and after experiencing both conditions: driving with and without the active massage system. One BMW 20-way comfort seat 
was equipped with the new pneumatic LWMAS. All participants were asked to drive 7 laps on a BMW closed test track of approximately $20 \mathrm{~km}$. The track was composed completely out of asphalt. The restrictions on the track were a maximum speed of 120 $\mathrm{km} / \mathrm{h}$ on straightaways and maximum speed of 80 $\mathrm{km} / \mathrm{h}$ in curves. Surface EMG was measured periodically during the entire test at the track with half the measurements with and without the LWMAS activated. The average duration of experiment 1 was 80 minutes. Surface EMG was recorded using 4 pairs of surface electrodes (Neuroline type AMBU 700 15-K; $20-\mathrm{mm}$ interelectrode distance) at the middle of the muscle bellies of both musculus trapezius pars ascendance and musculus rhomboideus. A withinsubject design was chosen. Recordings with and without an active LWMAS were taken of the same subject on the same day without replacing the electrodes. After completing the questionnaires, an experimenter discussed possible solutions for problems they identified like causes of discomfort experienced by the test subject. The test is more extensively described by Franz et al. [3].

Experiment 2: For this research study, pressure sensors were implanted through minimally invasive surgery with a canula into the middle of the intervertebral disc (nucleus pulposus) of one subject. The surgery was run the same way as an intervertebral disc operation using endoscope technology [8]. The sensor was implanted in the area of the spine where effects are expected in the disc: L5-S1. According to the ideal pressure distribution $[7,8]$ in this test car is $54.0 \%$ on the buttocks and $6.4 \%$ on the front thighs (= "IDEAL"). Two additional settings were produced: "MAX" with a maximal support of the frontal thighs $(10.6 \%)$ and a smaller load on the buttocks $(476 \%)$ and "MIN" with a minimal support of the frontal thighs $(0.4 \%)$ and more load on the buttocks $(66.5 \%)$. In all three situations the backrest position was kept constant in relation to the seat pan during the test procedure to avoid influence of the backrest angle on the load in the spine. The participant was also asked to give a short opinion on the discomfort feelings for each seat setting (MAX, MIN and IDEAL), without being informed which seat setting was currently used. A t-test for repeated measurements (within participant design) was carried out to check for significant differences $(\mathrm{p}<0.05)$. The test is more extensively described by Zenk et al. [8].

Experiment 3: Fifteen males and six females, participated in the experiment (height 1.78 (1.63 to 1.92) meters and weight $76 \mathrm{~kg}$ (48 to 107)). Three seats were used: one seat from a luxurious car, one from a sports car and the new concept seat based on the human body contour. All seats had the same backrest angle $\left(25^{\circ}\right)$ and seat angle $\left(14^{\circ}\right)$. The subjects had to sit on a covered car seat for several minutes while obtaining a driving position (see Figure 3). The seats were covered with a blanket to prevent that the subjects could estimate the branding of the seat. A sloping footrest to simulate the drivers' position was provided as well. It was not allowed for the participants to adjust the seat. They had to complete a questionnaire for each seat, which included questions whether they experience the seat as comfortable, luxurious, sporty, protected and relaxed. The keywords in this experiment were a selection from the descriptive words Zenk [7] found in his research on most important aspects for car seat users. At the end of the experiment an overall opinion was asked over the three seats. The test is more extensively described by Kamp et al. [4].

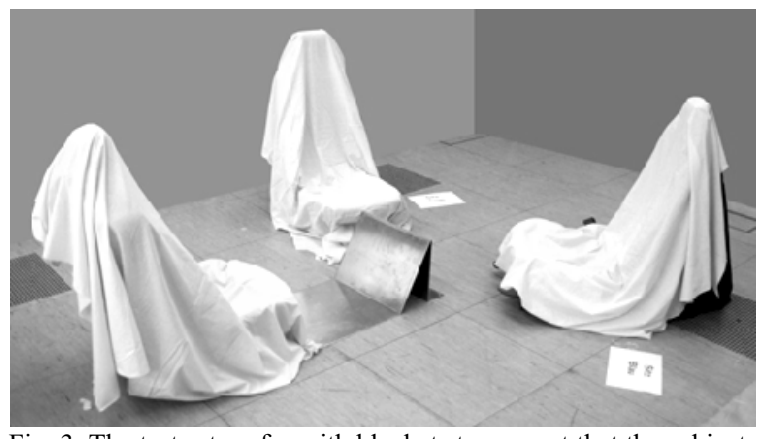

Fig. 3. The test set up for with blankets to prevent that the subjects see the chair.

\section{Results}

Experiment 1 'light weight massage': The EMG recordings showed that the trapezius muscle activity was significantly lower $(\mathrm{P}=.038)$ while driving with the active LWMAS than without it. Seventeen of the 24 subjects mentioned that their back felt relaxed while driving, and 18 felt relaxed after driving. No one felt that the driving behavior was disturbed by the LWMAS. However, one subject was distracted a bit by the EMG electrodes. Twenty-one participants liked the speed of the system, one felt the speed was too fast, and two felt it to be too slow. Twenty-three participants liked the intensity of the LWMAS, whereas one found the intensity to be too strong. The average comfort was also significantly improved.

Experiment 2 'effects in the body': The mean and maximum values of the intervertebral disc pressure 
for the three different seat settings are shown in Figure 4. In the MAX situation there is a maximum support of the frontal thighs and the mean value is 0.95 bar (SD during 15 seconds recording time is 0.08 ). The lowest pressure values are achieved in the IDEAL seat position. MIN (no support of the frontal thighs) resulted in the highest pressure values. The ttest analysis of the measurements showed that the lowest pressure values are significantly $(p<0.05)$ different in the "IDEAL" seat position compared with the others. The test participant was not informed of the seat setting, but felt that the "MAX" and "MIN" positions were less comfortable. It might be worthwhile to mention that these values are all far below the intervertebral disc pressure values recorded while sitting upright and standing [8].

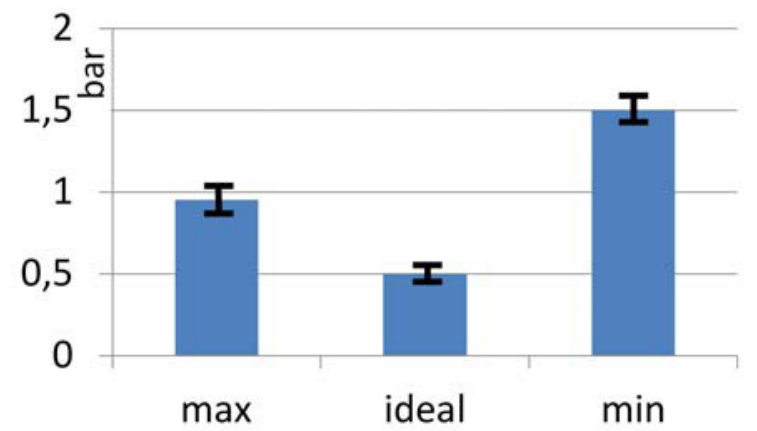

Fig. 4. The mean value of the disc pressure in the three conditions

Experiment 2 'effects in the body': The mean and maximum values of intervertebral disc pressure for the three different seat settings are shown in Figure 4. In the MAX situation there is a maximum support of the frontal thighs and the mean value is 0.95 bar (SD during 15 seconds recording time is 0.08 ). The lowest pressure values are achieved in the IDEAL seat position. MIN (no support of the frontal thighs) resulted in the highest pressure values. The t-test analysis of the measurements showed that the lowest pressure values are significantly $(\mathrm{p}<0.05)$ different in the "IDEAL" seat position compared with the others. The test participant was not informed of the seat setting, but felt that the "MAX" and "MIN" positions were less comfortable. All three positions were rated as comfortable.

Experiment 3: After all seats were tested, participants had to indicate which of the seats they thought was most comfortable, luxurious, sporty, protected and relaxed. Figure 5 shows the results of the words representing contours of each seat. The soft seat with wing was most frequently rated as luxurious and protected. The curved and hard seat was frequently rated as relaxed. If the participants are asked to indicate which seat they would choose for their own car on a scale from $1-10(1=$ very bad, $10=$ excellent $)$. The wings + soft seat received an average of 7.5 , the flat + hard 2 a 6.0 and the curved and hard a 6.8 .
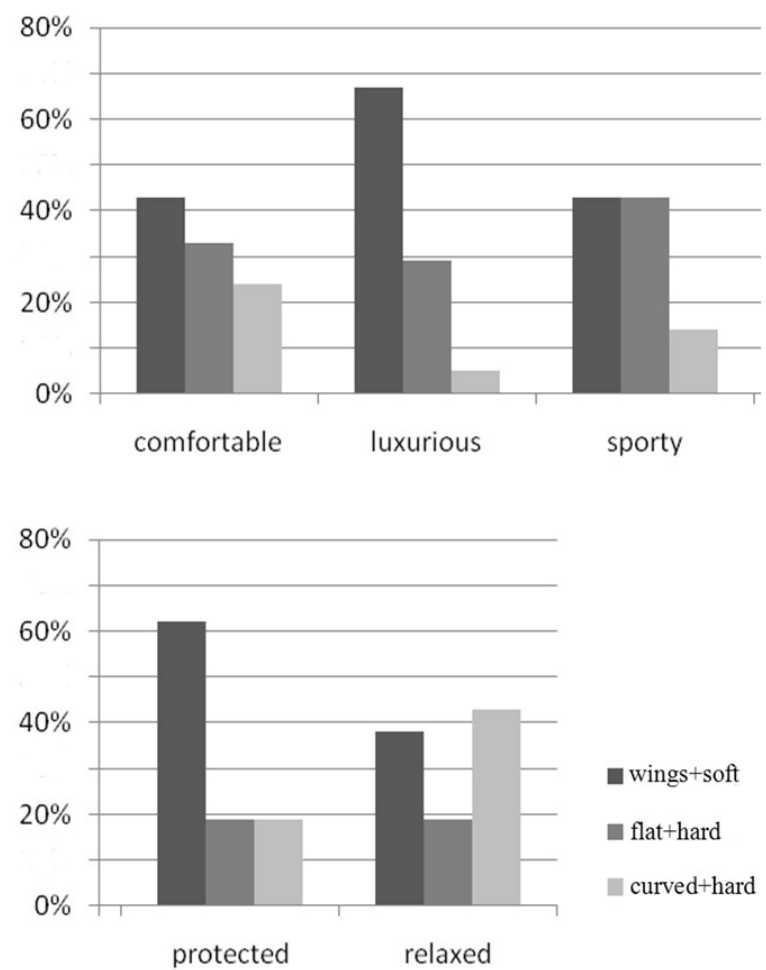

Fig. 5. The percentage of the subjects experiencing the seat a most comfortable, luxurious, sporty, protected and relaxed.

\section{Discussion}

The three experiments show that it is possible to replace current systems with light weight seats or parts of the seats and at the same time improve the comfort. The light weight massage system does reduce the weight of massage systems and is clearly noticed by the drivers. Also, the EMG signal is significantly reduced. An automatically adjustable seat can improve the comfort. Leads to measurable effects in the human body and does not need seat adjusting motors (experiment 2). Experiment 3 is the most extreme weight reduction, in the sense that the seat is completely new designed focused on light weight. There is no improvement of comfort experience recorded, but the experience is comparable with current seats. Optimizing this seat could improve the comfort 
and is a promising new way of designing seats for vehicles and will be applied in other vehicles as well.

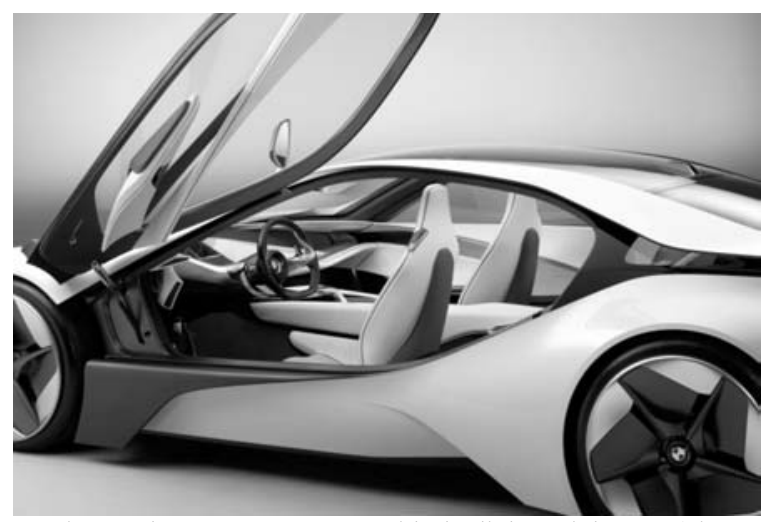

Fig. 6. The new concept car with the light weight seat (picture BMW)

The studies mentioned in this paper are only case studies and it is difficult to draw general conclusions. However, the more extensive studies in this area $[1,2$, $3,4,8]$ show similar results. The first experiments with lightweight seats and seat elements show that the comfort can be kept at the same level and can even improve if new technologies, designs and materials are used. Based on this research the new composite seat with small inflatable cushions and a 3 mesh layer (making airflow between body and seat possible) will be developed further. The first version (see Figure 6) is shown in the BMW new concept car of
2010 [1]. However, this concerns only the first steps in this new area of research within 'sustainable ergonomics'. For defining a general model for the optimum between lightweight and comfort more solid research and new models will be needed.

\section{References}

[1] M. Franz, Comfort, experience, physiology and car seat innovation, Ph.D. Dissertation, Delft University of Technology, 2010 .

[2] M. Franz, I. Kamp, A. Durt, Ü. Kililncsoy, H. Bubb, P. Vink, A lightweight car seat shaped by human body contour. Int $\mathrm{J}$ of human factors modeling and simulation 2012, in press.

[3] M. Franz, R. Zenk, P. Vink, S. Hallbeck, The effect of a light weight massage system in a car seat on comfort and electromyogram. J Manipulative and Physiological Therapeutics 34 (2011), 107-113.

[4] I. Kamp, The influence of car seat design on its character experience. Applied Ergonomics (2011) in press

[5] G. Kyung, M.A. Nussbaum. Driving sitting comfort and discomfort (part II): relationships with and prediction from interface pressure. Int J Ind Ergon 28(2008),526-538.

[6] P. Vink, S. Hallbeck, editorial: comfort and discomfort studies demonstrate the need for a new model. Applied Ergonomics (2011) in press.

[7] R. Zenk, Objektivierung des Sitzkomforts und seiner automatischen Anpassung, Ph.D. Dissertation, München University of Technology, 2008.

[8] R. Zenk, M. Franz, H. Bubb, P. Vink. Technical note: spine loading in automotive seating. Applied Ergonomics (2011) in press 